\title{
Comunicación web y análisis económico financiero en el sector del transporte: Caso Busmet
}

\section{Web communication and economic analysis in the}

transport sector. The case of BUSMET
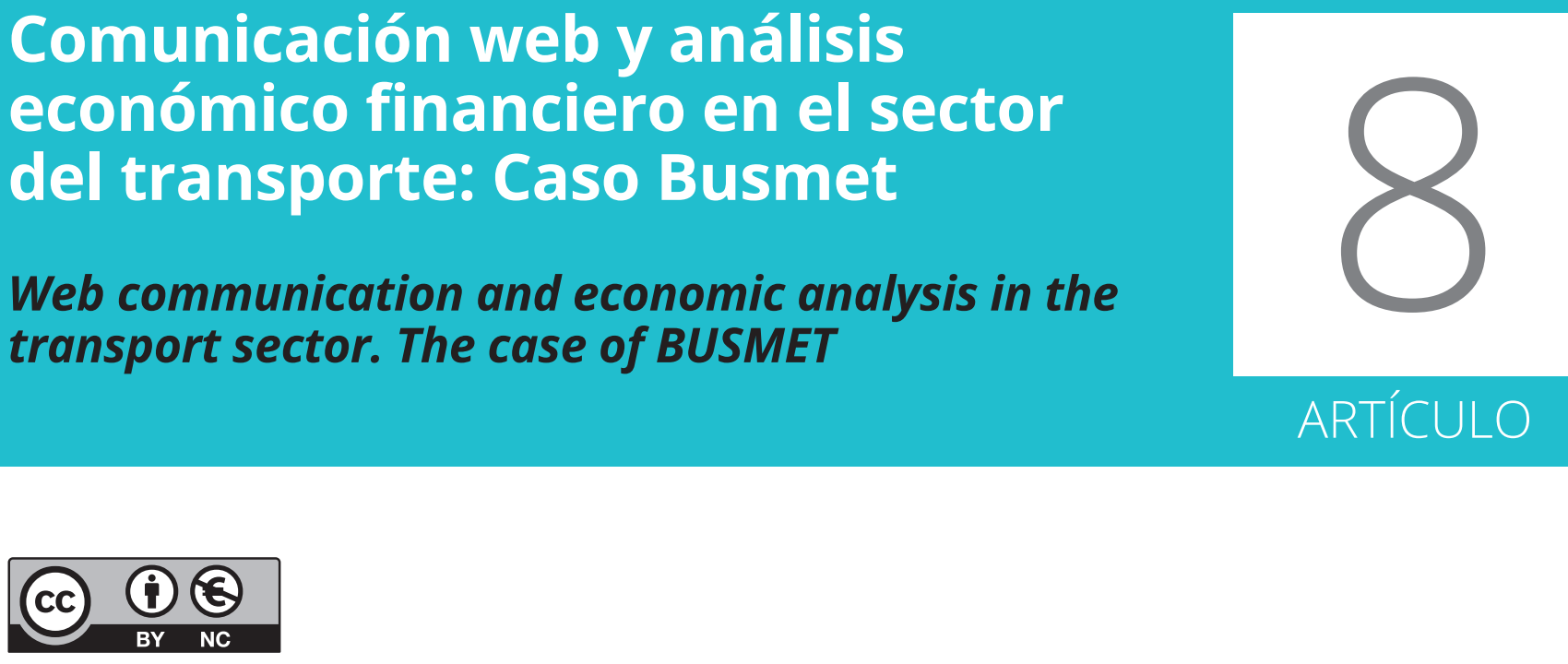

\section{Nuria Arimany Serrat}

Universidad de Vic-Universidad Central de Catalunya

Doctora en Ciencias Económicas y Empresariales por la Universidad de Barcelona. Profesora titular de la Universidad de Vic-Universidad Central de Catalunya (Uvic-UCC) con trayectoria docente en contabilidad y finanzas. Actualmente coordina un grupo de investigación sobre emprendimiento e innovación (Uvic-UCC), reconocido por la Generalitat de Catalunya.

nuria.arimany@uvic.cat

ORCID: 0000-0003-0323-6601

\section{Clara Eugenia de Uribe-Salazar Gil}

Universidad de Vic-Universidad Central de Catalunya

Doctora en Publicidad y Relaciones Públicas por la Universidad Autònoma de Barcelona. Master en Community Management y Dirección de Redes Sociales, por la Universidad de Alicante. Actualmente imparte docencia en la Universidad de Vic-Universidad Central de Catalunya, en la Escuela Superior de Relaciones Públicas (UB) y en Educación Online (UOC). Consultora asociada de Gabinete Uribe.

clara.uribe@uvic.cat

ORCID: 0000-0003-1363-9033 


\section{Anna Sabata-Aliberch}

Universidad de Vic-Universidad Central de Catalunya

Doctora por la Universidad de Barcelona (UB). Profesora de Contabilidad de Costes y Empresa en el Departamento de Economía y Empresa de la Universidad de Vic- Universidad Central de Catalunya. Miembro del grupo de Investigación "Empren" (Uvic- UCC). Autora de comunicaciones en diversos congresos vinculados a la Contabilidad de Costes en el Sector primario y a la Responsabilidad Social Corporativa.

annam.sabata@uvic.cat

ORCID: 0000-0003-4999-4360

Fecha de recepción: 30 de noviembre de 2017 / Aceptación: 14 de marzo de 2018

\section{Resumen}

El objetivo de este estudio consiste en realizar un análisis de la situación actual del sector del transporte de viajeros por autobús, en la zona metropolitana de Barcelona, mediante el estudio de la realidad económica financiera, en el período 2008-2016, de BUSMET y de su actual transparencia web. Los resultados evidencian la supervivencia de las empresas integrantes por sus correctos indicadores económicos y financieros en el período 2008-2016, junto con la adecuada transparencia en su comunicación web, aunque debe mejorar su comunicación en RSC.

\section{PALABRAS CLAVE}

Transporte de viajeros, Análisis económico-financiero, Transparencia web, Comunicación, RSC.

\begin{abstract}
We carried out an analysis of the current situation of bus passenger transport in the Metropolitan Area of Barcelona, through the study of economic and financial BUSMET data for the 2008-2016 period, and the current degree of web transparency. The results indicate the viability of the constituent companies as shown by satisfactory economic and financial indicators in the 2008-2016 period, together with sufficient web transparency in gene$\mathrm{ral}$, though there is room for improvement in communication of CSR initiatives.
\end{abstract}

\section{KEYWORDS}

Transport of travellers, Economic-financial performance, Transparency web, Communication, CSR.

\section{INTRODUÇÃO}

El sector transporte, de gran importancia en la actualidad ya que repercute directamente en el crecimiento económico y en la creación de empleo, está sujeto a importantes retos. Los operadores privados, que evolucionan con nuevas fórmulas de integración, presentan aceptables indicadores económicos y financieros y muestran un interés creciente por la mejora de la sostenibilidad del sector y la transparencia del mismo, tal como evidencia el caso BUSMET objeto de estudio. 
De otra parte, un elemento esencial del sector es conseguir la reducción de las emisiones de cO2 que desencadena el mismo, en sintonía con la sostenibilidad que contempla la Responsabilidad Social Corporativa (RSC).

El objetivo de este estudio consiste en realizar una panorámica de la situación actual del sector del transporte de viajeros en autobús, en la zona metropolitana de Barcelona, mediante un mayor conocimiento de su realidad a nivel económico y financiero en el período 2008-2016 y un mayor conocimiento de su transparencia web, visibilizando un caso concreto, el caso BUSMET, para avalar este análisis.

La metodología, después de caracterizar el sector objeto de estudio, y de la oportuna revisión de la literatura, es exploratoria, y se exploran dos ámbitos: la salud económica financiera de las 30 empresas integrantes de BUSMET en el período 2008-2016 y el análisis de su transparencia web a través de diferentes ítems.

Finalmente, se presentan los resultados y las conclusiones del estudio incidiendo en la salud económica y financiera de las 30 empresas integrantes de BUSMET y su grado de transparencia web (especialmente en referencia a la Responsabilidad Social Corporativa).

\section{SECTOR DEL TRANSPORTE, SOSTENIBILIDAD Y TRANSPARENCIA}

El sector del transporte de la UE depende mayoritariamente de los combustibles fósiles, los carburantes derivados del petróleo representan el 96\% del suministro energético siendo el transporte por carretera (de mercancías y personas) el medio que utiliza la mayor parte de la energía.
El sector del transporte, cuyas emisiones de dióxido de carbono representen alrededor del $20 \%$ del total de emisiones de gases de efecto invernadero de la $U E$, debe reducir sus emisiones en un 60\% hasta 2050 para cumplir los objetivos sobre el cambio climático de acuerdo con la Conferencia sobre el Cambio Climático de París en 2015. Cabe destacar, que las emisiones de $\mathrm{CO} 2$ (principal gas ligado al efecto invernadero) están creciendo notablemente y el transporte es el sector en el que crece con más intensidad y es el transporte por carretera, el medio de transporte más extendido y el que más contamina. Según datos de la Comisión Europea, produce alrededor del 70\% de todas las emisiones de dióxido de carbono derivadas del transporte.

Los costes externos del transporte son los efectos negativos del transporte que no se internalizan en el precio pagado por el usuario, como la contaminación del aire, los accidentes y la congestión. Estos costes no se pueden ignorar, ya que, generan costes reales para la sociedad, como el cambio climático, los problemas de salud y las retenciones. Si se monetizan estos costes para los usuarios, operadores e inversores, tomarán las decisiones más apropiadas y más sostenibles desde el punto de vista de la sociedad en aspectos relacionados con el modo de viaje, el despliegue de nuevas tecnologías y el desarrollo de infraestructuras.

Por todo ello, buscar la sostenibilidad va a transformar el sector logístico, trasformando modelos de negocio, tecnologías utilizadas, gestión de las emisiones de CO2 (Ciliberti et al, 2008). Además, el 63\% de los clientes cree que la logística se convertirá en un factor estratégico para impulsar la disminución del $\mathrm{CO} 2$ y junto con la importancia económica que ya tiene en la actualidad, la logística será un elemento esencial para conseguir la reducción de las emisiones de CO2 en la economía global 
(Diane et al, 2010). El estudio también apunta que se pueden lograr reducciones significativas de $\mathrm{CO} 2$ en el sector logístico sin tener que esperar a que aparezcan grandes avances tecnológicos.

Con todo, cualquier empresa relacionada con el transporte de pasajeros, como en el caso de BUSMET, debe tener presente la Responsabilidad Social Corporativa (RSC), especialmente en el aspecto medioambiental, y debe comunicar de forma transparente sus acciones en las páginas web.

El transporte urbano de viajeros constituye uno de los ámbitos más importantes desde el punto de vista de la movilidad diaria de personas, en concreto, en el año 2014 se realizaron en España un total de 1.631 millones de viajes en autobús en entornos urbanos y metropolitanos (MFOM, 2014), de los cuales aproximadamente la mitad corresponden a las cinco ciudades con mayor población: Madrid, BarceIona, Valencia, Sevilla y Zaragoza. La evolución de este sector de transporte a lo largo de los últimos años muestra los efectos que la crisis económica global ha tenido sobre la movilidad de las personas en las ciudades: el número de desplazamientos ha descendido aproximadamente un 12\% respecto al año 2007, siendo este descenso más acusado en las principales áreas metropolitanas que en el resto de ciudades españolas. A pesar de este descenso en la demanda de transporte por autobús, que cabe atribuir al ciclo macroeconómico recesivo y que también se detecta en el uso del metro, puede esperarse que la recuperación de la economía volverá a hacer crecer las tasas de utilización del transporte público urbano a ritmos elevados en un futuro próximo. Además, el uso del automóvil privado para realizar desplazamientos urbanos va a verse sometido cada vez más a restricciones motivadas por los problemas de congestión y las emisiones contaminantes, que provocan un deterioro de la salud de los habitantes. Por tanto, la sostenibilidad y su transparencia deben estar presentes en las empresas de transporte de pasajeros.

En concreto, los periodos punta de contaminación que han empezado a sufrir las grandes ciudades en España han llevado a las administraciones locales de Madrid y Barcelona a introducir restricciones y mecanismos más estrictos de control del tráfico (prohibiciones de aparcamiento, circulación limitada o restringida por zonas, uso de peajes, etc.)

Ante esta situación, es predecible a lo largo de los próximos años un incremento en la utilización de las redes de transporte público: autobuses urbanos y metropolitanos, metro y ferrocarril de cercanías, junto a la creciente demanda de infraestructuras adecuadas para la utilización de la bicicleta como un modo de transporte generalizado para los desplazamientos habituales por motivos no vinculados a actividades meramente deportivas o de ocio. En este contexto, resulta de interés tanto desde un punto de vista puramente académico como para el diseño de políticas de transporte, disponer de información de los resultados actuales que están obteniendo el conjunto de empresas públicas y privadas que se encargan de la provisión de los servicios de transporte urbanos de autobús.

A pesar de su enorme relevancia cuantitativa para la movilidad en las ciudades, y del elevado volumen de recursos públicos que absorben las subvenciones públicas destinadas a reducir los precios y promover la utilización de los autobuses urbanos, existe una literatura muy escasa en España que tenga por objeto el análisis en el subsector de los autobuses urbanos (De Rus y Nombela, 1997; Martín Urbano et al., 2012). Por todo ello, este trabajo se centra en evidenciar el modelo BUSMET, la situación eco- 
nómica y financiera de las empresas implicadas y su comunicación web, a nivel de transparencia, especialmente en la variable de RSC.

Este estudio pretende realizar una panorámica de la situación actual del sector del transporte de viajeros en la zona metropolitana de BarceIona, con un mayor conocimiento de su realidad con el análisis económico y financiero en el período 2008-2016 y su transparencia web en el caso BUSMET.

\section{REVISIÓN DE LA LITERATURA}

Las instituciones públicas o privadas deben conocer las demandas sociales para conseguir un desarrollo sostenible con efectos a largo plazo (Robbins,1994; Porter y Kramer, 2003) y las empresas del transporte no son una excepción. Además, la sostenibilidad y la transparencia refuerza la posición competitiva y la mayor aceptación de las empresas (De la Cuesta, 2006) minimizando el riesgo reputacional, con ventajas competitivas (Rodríguez, Ricart y Sánchez, 2001), por lo que interesa su trasparencia informativa, comunicativa y de RSC.

Con el desarrollo de internet y de las webs corporativas, la página web ayuda a reflejar la transparencia de las empresas en cuentas y en comunicación (Cameron, 2004; Meijer,2007; Tagesson, Blank, Broberg y Collin, 2009), además la percepción de los usuarios sobre acciones de RSC puede influir en la cuota de mercado que consiguen las empresas (Currás, Bigne, y Alvarado, 2009). Todas las decisiones en RSC suponen repercusiones en el medioambiente y una postura proactiva (especialmente en el sector del transporte), es valorada positivamente por la sociedad (Piñeiro, Quintas y Caballero 2009). Por tanto, el análisis de la comunicación web es crucial para evidenciar la transparencia y sostenibilidad empresarial. De otra parte, una adecuada gestión y comunicación web mejora la imagen (Saxton y Dollinger,2004; Rindova, Wiliiamson, Petkova y Sever, 2005; Rodríguez y García de Madariaga, 2011). El sector del transporte urbano tiene una destacada importancia en la sociedad, por lo que, conocer su salud económica financiera y su comunicación web permite evidenciar su potencial supervivencia.

Tal como describe Jonathan, en 2002, el éxito del web se asocia al contenido web (cantidad de información, variedad) y a la navegación web (organización, estructura, composición y secuenciación). Desde la perspectiva del usuario se debe tener en cuenta la usabilidad (Araque \& Montero, 2006), basada en factores como la velocidad de acceso, la interactividad, y la capacidad de respuesta. Además, una utilización sencilla del web crea una imagen positiva (Huertas Roig i Xifra Triadú, 2009), y para el caso de la transparencia en RSC en el transporte aborda una problemática medioambiental cada día más patente en nuestra sociedad.

\section{METODOLOGÍA}

La metodología, después de caracterizar el sector objeto de estudio, y de la oportuna revisión de la literatura, es exploratoria, y se exploran dos ámbitos:

1. El análisis económico financiero mediante diferentes indicadores financieros utilizados en una muestra de 30 empresas, participantes en BUSMET, extraída de la base de datos SABI en el período 2008-2016.

La información cuantitativa de estas 30 empresas se ha obtenido de la base a los datos SABI (Sistema de Análisis de Balance Ibérico). Para cada empresa analizada se ha tenido en cuenta sus estados financieros y los indicadores económicos y financieros que permiten deter- 
minar su salud económica y financiera (solvencia a corto plazo, endeudamiento, rentabilidad económica y financiera y capitalización) para una comprensión general y aproximada de la situación global del grupo de empresas analizadas en el período objeto de estudio.

2. El análisis web de diferentes ítems, utilizados académicamente, para valorar la trasparencia web de las 30 empresas participantes en BUSMET.

Para el análisis de la comunicación de la RSC en la muestra objeto de estudio de las 30 websites corporativas (reducidas a 20 webs por la integración de empresas BUSMET en una misma web) se ha preparado una ficha técnica de recogida y sistematización de la información cualitativa, validada académicamente, estructurada en dos áreas principales: Contenido y Navegabilidad. Ambas áreas definen la comunicación web de la RSC e identificana las empresas de Busmet que desarrollan las mejores prácticas.

- En referencia al Análisis de Contenido de la RSC en las páginas web, se han tenido en cuenta variables que corresponden a la sección "about us", así como un apartado específico sobre RSC. La visión desde un ángulo cualitativo nos ofrece informaciones a través del estudio de las páginas web de las empresas que forman Busmet, y nos da una perspectiva general de las tendencias de comunicación de la RSC. De su análisis se dislumbran los principales elementos de la RSC en las empresas de Busmet.

- Respecto a la Navegabilidad de las páginas web, se han determinado como variables relevantes el Mapa web y el buscador interno:

MAPA WEB - Lay out (puntuación: 1=no disponible; 2= Se indica Ruta de navegación; 3= Mapa web en la página principal; 4= Ambas aplicaciones siempre visibles; $5=$ disponible en Lay out).

MAPA WEB - Información de Interés y Actualización (Contenidos temporales o de frecuente actualización: eventos, noticias, artículos).

MAPA WEB - Elementos simples de interactividad (disponibilidad FAQS, utilización reciente FAQS - Comentarios (fecha último mensaje, respuesta de la empresa) -

Ayuda al Usuario. Con la puntuación Si=1 y $\mathrm{No}=0$.

MAPA WEB -Otros de Interés (Diseño Web Compatible con otros sistemas de navegación: Ipod, smartphones, tablets, etc.). Con la puntuación $\mathrm{Si}=1$ y No=0.

La recogida de información de las páginas web y de la información de la base de datos SABI se realizó el día 8 de enero de 2018.

Finalmente, se presentan los resultados y las conclusiones del estudio para poder valorar la salud económica y financiera BUSMET y su transparencia web, así como su RSC, en las 30 empresas integrantes de BUSMET.

\section{CASO BUSMET}

Actualmente, las empresas del sector del transporte y la logística son fundamentales para el desarrollo de la sociedad y de la economía, además el transporte público de viajeros, facilita la comunicación y la vida entre comunidades, así por ejemplo, el transporte público facilita que muchos ciudadanos estudien, trabajen, se relacionen o sencillamente se puedan desplazar en busca de oportunidades en zonas metropolitanas, como por ejemplo, en el caso de la Área Metropolitana de Barcelona, en diferentes municipios como: Hospitalet, BadaIona, Santa Coloma de Gramenet y Sant Adrià 
de Besós; en diferentes comarcas como el Baix Llobregat, el Maresme, el Vallès Occidental, el Oriental, el Garraf o en diferentes ciudades medianas del interior.

Para la eficiencia de este transporte público se precisan redes que combinen o intercambien modos de transporte (autobús, metro, ferrocarril o tranvía). En el Área Metropolitana de Barcelona, esta red multimodal está operada por diferentes empresas, algunas de titularidad pública, privada y otras concesionadas, además se suele subvencionar el precio del billete para que sea asequible al público en general mediante contratos-programa para mejorar la calidad del servicio prestado a los ciudadanos, con la renovación de flotas de vehículos, mejorando las frecuencias de paso, el tiempo de espera, acondicionando estaciones, marquesinas de espera, etc.

El acceso a las subvenciones depende de la viabilidad de las empresas de transporte de viajeros y sin estas subvenciones muchas compañías no pueden operar. En concreto, de las 25 empresas que operaban en el transporte público de viajeros, en el año 1999, en el área metropolitana de Barcelona, sólo 3 tenían subvención (por su carácter público), las 22 restantes operadoras que precisaban renovar o modernizar sus flotas, ampliar franjas horarias de ruta, entre otras necesidades, no conseguían las subvenciones, y aunque estaban agrupados en la FECAV (Federación Catalana de Autotransporte de Viajeros), no conseguían influir en la Administración para acceder a los contratos-programa del Estado y, por tanto, a las subvenciones.

En consecuencia, los directivos de FECAV optaron por mejorar su comunicación, previa cohesión interna entre las 22 compañías (cohesión de grupos), con el objetivo de unirse bajo un mismo nombre y no desaparecer del mapa, na- ciendo la marca BUSMET que integraba las 22 operadoras.

En ese momento, se diseñó un programa de identidad visual, con máxima difusión, y su logotipo se aplicó en títulos de transporte, en paneles informativos de las marquesinas y paradas, en rótulos de identificación de los vehículos de cada compañía, en trípticos informativos, en folletos horarios y en todos aquellos documentos comunes.

Seguidamente, se definieron los objetivos a alcanzar: participar en el contrato-programa; recuperar e incrementar la cuota de mercado; renovar flotas, paradas; implantar máquinas validadoras de títulos; obtener la certificación de calidad (ISO 9002); en definitiva, prestar un mejor servicio y fidelizar a los pasajeros.

Posteriormente, se realizó la adecuada comunicación interna entre los trabajadores de la marca BUSMET, con una descripción clara de los objetivos de BUSMET, para implicar en el proyecto a los trabajadores que no querían perder su puesto de trabajo. Todo ello, con voluntad de cohesionar el equipo.

Una vez cohesionado el equipo, se creó un plan de comunicación externa, con un eslogan identificativo, para visualizar el transporte de proximidad de personas mediante las $22 \mathrm{em}$ presas integrantes en BUSMET, para plantear la participación de BUSMET en el contrato programa. Este planteamiento era más lógico ya que se interactuaba con un solo interlocutor (BUSMET).

La presentación pública de BUSMET, a través de los medios de comunicación; el programa lobbying (para remarcar la importancia y trascendencia de los operadores privados -con una situación económica crítica en ese momento- en el transporte público de viajeros en toda el Área Metropolitana de Barcelona, al 
unir 164 municipios) y la búsqueda de acuerdos de consenso con las Administraciones para comprender la situación, promovieron algunas importantes ayudas vía subvenciones.

La presentación pública de BUSMET se favoreció por la coincidencia temporal de la concienciación llevada a cabo en Barcelona, Cataluña y España, de dejar el coche y apostar por el transporte público para reducir las emisiones de los gases efecto invernadero para mejorar el medioambiente.

Después de este proceso, BUSMET se posicionó como un actor de referencia en el transporte público de viajeros por carretera en el Área Metropolitana de Barcelona, con una imagen y una identidad propia, bien valorada por los diferentes públicos, con objetivos consensuados y una marca reconocida en las Administraciones Públicas, que permitió conseguir subvenciones, acceder a la integración tarifaria y aumentar la cuota de mercado.

BUSMET arrancó en 1999, y en 2002 tres años después de la puesta en marcha del plan estratégico de comunicación, el cierre del ejercicio económico, reflejaba un incremento de cuota de mercado del 6,47\% para las empresas que integraban BUSMET.

Hasta a día de hoy, enero de 2018, sigue siendo un modelo de éxito, por la cohesión, las actuaciones conjuntas de las empresas integrantes y las múltiples operaciones mercantiles entre ellas, que han propiciado que BUSMET esté integrada por 30 empresas actualmente (tabla 1).
Tabla 1. Empresas integrantes de BUSMET

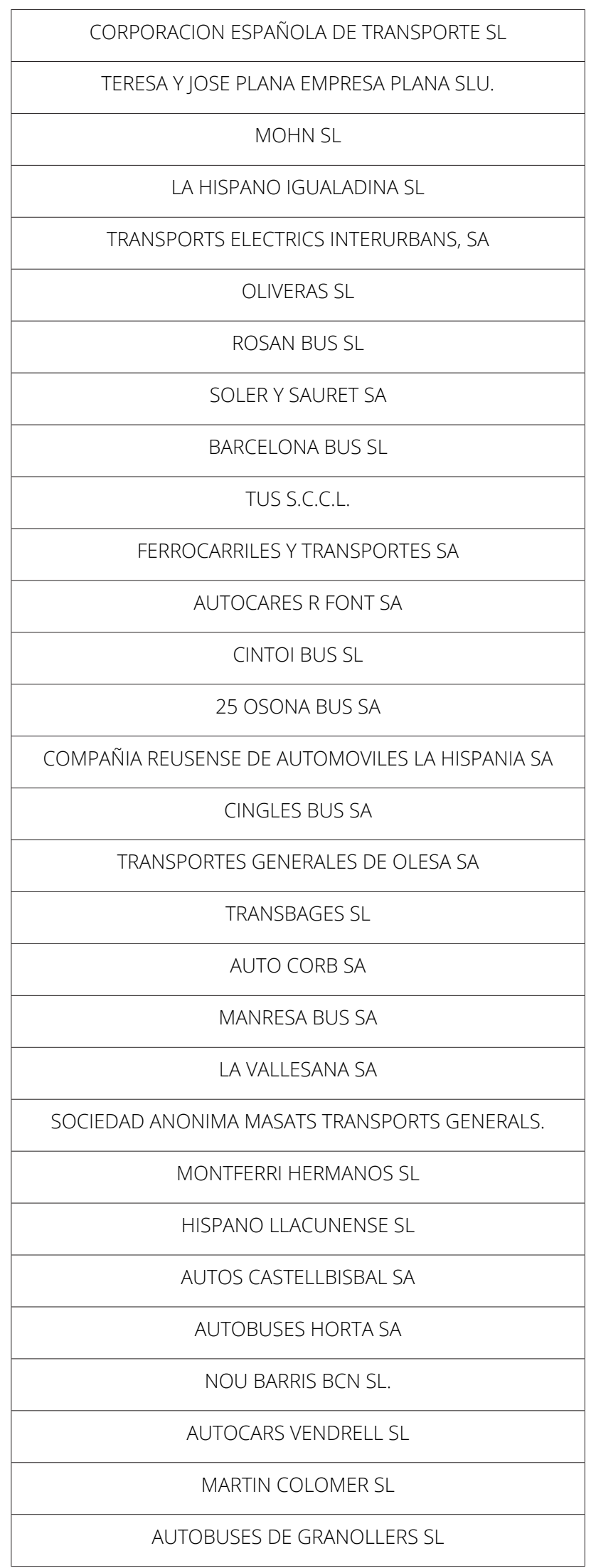




\subsection{ANÁLISIS ECONÓMICO FINANCIERO DE LAS EMPRESAS INTEGRANTES DE BUSMET}

Para realizar un análisis de la situación económica y financiera de las empresas integrantes en BUSMET en el período 2008-2016, se han calculado los principales indicadores financieros, partiendo de un análisis patrimonial previo.

En concreto, para el análisis de la situación financiera a corto plazo, que consiste en determinar la capacidad que tienen las empresas para hacer frente a sus obligaciones de pago a corto plazo, se calcula: el fondo de maniobra (activo corriente menos pasivo corriente) y la solvencia a corto plazo (activo corriente/pasivo corriente). Además, se complementa el estudio con el análisis de los flujos de efectivo de la actividad de explotación de las empresas integrantes en la marca BUSMET.

Para el análisis de la situación financiera a largo plazo, cuyo objetivo principal es medir la capacidad que tienen las operadoras para satisfacer las deudas a largo plazo, se utilizan como principales indicadores el endeudamiento (total deudas/patrimonio neto y pasivo) y la calidad de la deuda (deudas corto plazo/total deudas). También se valora si el crecimiento de las operadoras es equilibrado, según criterio de Amat y Perramon (2012), es decir, si las operadoras presentan una gestión eficiente de activos ( $\triangle$ Ventas $>\triangle$ Activos); una gestión financiera prudente ( $\triangle$ Activos $>\triangle$ Deudas) y una buena gestión de gastos ( $\triangle$ Resultados $>\Delta V$ Ventas).

Respecto al análisis económico, que tiene por objetivo el estudio de los resultados con impacto en la empresa, y en explicar cómo se han producido los mismos, identificando las causas que han provocado la variación de estos resultados en el periodo considerado, se utilizan los siguientes indicadores: porcentaje horizontal de los ingresos de explotación; porcentaje horizontal del resultado neto; valor añadido (ingresos de explotación menos gastos de explotación) que al dividirlo por los gastos de personal nos da la productividad del personal ;rentabilidad financiera (ROE = resultado neto/ patrimonio neto-resultado neto) y rentabilidad económica (ROA = BAIT/activo).

Finalmente, el análisis patrimonial refleja el aumento del patrimonio neto (capitalización) en el período objeto de estudio; o bien, la disminución del patrimonio neto (descapitalización) en el período analizado de 2008-2016. Es decir, el aumento o disminución de la riqueza y garantías de las empresas integrantes de la marca BUSMET.

\subsection{ANÁLISIS \\ PATRIMONIAL PREVIO}

En el análisis patrimonial previo se analizan las medias de las masas patrimoniales del balance de situación de las 30 empresas integrantes de BUSMET en el período 2008-2016. Para valorar si presentan equilibrio patrimonial, es decir, si todas las inversiones en inmovilizado están financiadas por capitales permanentes (patrimonio neto y pasivos a largo plazo). Analizando las empresas objeto de estudio, se constata que gozan de un fondo de maniobra positivo (activo corriente menos pasivo corriente) y que sus activos no corrientes o inmovilizados (de un 67,51\%) están financiados por capitales permanentes $(78,13 \%)$, identificando una situación normal de equilibrio de las masas patrimoniales (figura 1) 


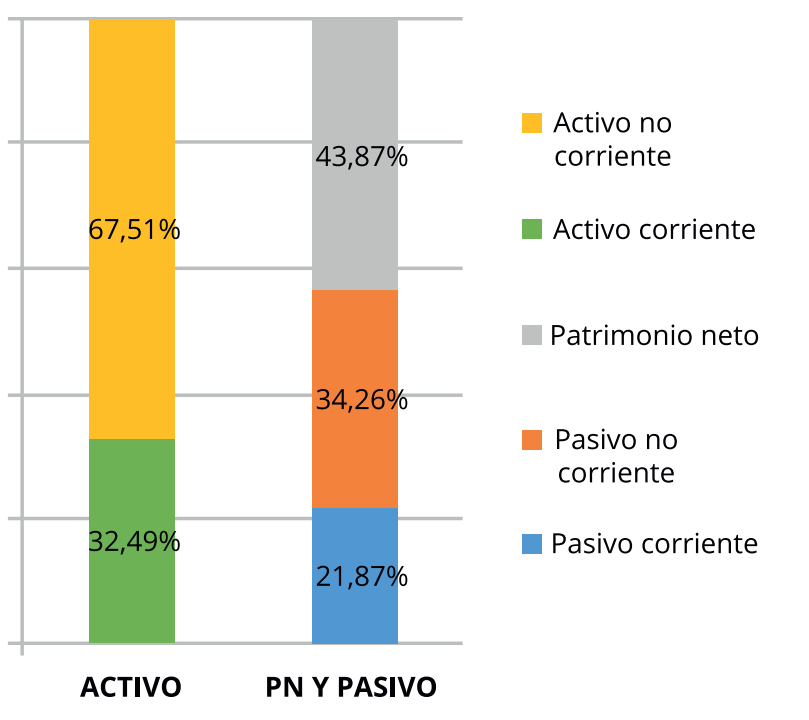

Figura 1. Estructura económica y financiera de las empresas integrantes de Busmet

Tabla 2. Liquidez de las empresas integrantes de Busmet

\begin{tabular}{|c|c|c|c|c|c|c|c|c|c|c|}
\hline MILES DE EUROS & 2008 & 2009 & 2010 & 2011 & 2012 & 2013 & 2014 & 2015 & 2016 \\
\hline Fondo de maniobra & 634,74 & 751,63 & 986,87 & $1.641,82$ & $1.176,39$ & $1.260,50$ & $1.670,05$ & 747,97 & $1.532,48$ \\
\hline Solvencia corto plazo & 1,26 & 1,29 & 1,36 & 1,65 & 1,53 & 1,58 & 1,78 & 1,27 & 1,88 \\
\hline
\end{tabular}

Tabla 3. Flujos de Efectivo de las empresas integrantes de Busmet

\begin{tabular}{|c|c|c|c|c|c|c|c|c|c|}
\hline $\begin{array}{l}\text { ESTADO DE FLUJOS DE } \\
\text { EFECTIVO (miles de euros) }\end{array}$ & 2008 & 2009 & 2010 & 2011 & 2012 & 2013 & 2014 & 2015 & 2016 \\
\hline $\begin{array}{c}\text { A) FLUJOS DE EFECTIVO DE } \\
\text { LAS ACTIVIDADES DE EXPLO- } \\
\text { TACIÓN }\end{array}$ & (498) & 1713 & 2092 & 300 & 3437 & 1655 & 2921 & 3582 & 1549 \\
\hline $\begin{array}{l}\text { B) FLUJOS DE EFECTIVO DE } \\
\text { LAS ACTIVIDADES DE INVER- } \\
\text { SIÓN }\end{array}$ & (541) & (1341) & (2162) & (627) & (1948) & (1312) & (2583) & (1203) & (1963) \\
\hline $\begin{array}{c}\text { C) FLUJOS DE EFECTIVO DE } \\
\text { LAS ACTIVIDADES DE FINAN- } \\
\text { CIACIÓN }\end{array}$ & 1151 & $(273)$ & 236 & 121 & (1113) & (351) & 980 & (1514) & (323) \\
\hline
\end{tabular}

Tabla 4. Endeudamiento de las empresas integrantes de Busmet

\begin{tabular}{|c|c|c|c|c|c|c|c|c|c|}
\hline$\%$ & 2008 & 2009 & 2010 & 2011 & 2012 & 2013 & 2014 & 2015 & 2016 \\
\hline Endeudamiento & $69,05 \%$ & $65,59 \%$ & $63,07 \%$ & $60,09 \%$ & $55,22 \%$ & $52,22 \%$ & $51,50 \%$ & $47,23 \%$ & $40,38 \%$ \\
\hline Calidad de la deuda & $37,01 \%$ & $36,45 \%$ & $38,29 \%$ & $36,94 \%$ & $37,30 \%$ & $37,78 \%$ & $34,16 \%$ & $47,78 \%$ & $52,33 \%$ \\
\hline
\end{tabular}


Tabla 5. Crecimiento equilibrado de las empresas integrantes de Busmet. El símbolo $\triangle$, significa crecimiento

\begin{tabular}{|c|c|c|c|c|c|c|}
\hline & \multicolumn{2}{|c|}{$\begin{array}{c}\text { Gestión eficiente de los } \\
\text { activos }\end{array}$} & \multicolumn{2}{c|}{ Gestión financiera prudente } & \multicolumn{2}{c|}{ Gestión de los gastos } \\
\cline { 2 - 6 } & $\Delta$ Ventas & $\Delta$ Activos & $\Delta$ Activos & $\Delta$ Deudas & $\Delta$ Resultados & $\Delta$ Ventas \\
\hline $\begin{array}{c}\text { Crecimiento equili- } \\
\text { brado }\end{array}$ & $-7,573 \%$ & $-12,214 \%$ & $-12,214 \%$ & $-48,659 \%$ & $460,856 \%$ & $0,425 \%$ \\
\hline
\end{tabular}

\subsection{ANÁLISIS DE LA SITUACIÓN FINANCIERA A CORTO PLAZO DE BUSMET}

En referencia a la solvencia a corto plazo, que mide la capacidad de las empresas para hacer frente a las deudas a corto plazo mediante el activo corriente, en los ejercicios analizados se detecta una situación buena ya que en todos los ejercicios el fondo de maniobra es positivo y la ratio de solvencia a corto presenta valores comprendidos en la franja óptima $(1,5-2)$ en la mayoría de ejercicios (tabla 2). Es decir, las empresas integrantes de BUSMET presentan una buena capacidad para atender los compromisos de pago a corto plazo y los flujos de efectivo de la actividad de explotación, reflejados en el documento contable del Estado de Flujos de Efectivo, son positivos en todo el período considerado, corroborando una buena liquidez para estas empresas (tabla 3)

\subsection{ANÁLISIS DE LA SITUACIÓN FINANCIERA A LARGO PLAZO DE BUSMET}

En cuanto al endeudamiento (pasivo entre patrimonio neto y pasivo), se trata de empresas con un nivel de endeudamiento aceptable a partir de 2011, incluso ligeramente bajo en los dos últimos ejercicios, ya que el endeudamiento normal se considera entre un $40 \%$ y $50 \%$. Respecto a la calidad de la deuda (relación entre deudas a corto plazo respecto al total deudas), que significa que hay más deudas a largo plazo que a corto plazo, en las empresas integrantes de BUSMET la calidad de la deuda es buena ya que hay más deudas a largo plazo que a corto y en el último ejercicio, hay prácticamente las mismas deudas a corto plazo que a largo plazo (tabla 4).

En cuanto, al criterio aportado por Amat y Perramon (2012), en este periodo analizado hay una gestión eficiente de los activos ( $\triangle$ Ventas $>\triangle$ Activos); una gestión financiera prudente ( $\triangle$ Activos $>\triangle$ Deudas); y una buena gestión de los gastos ( $\triangle$ Resultados $>\Delta V$ Ventas). Por tanto, las empresas integrantes de BUSMET presentan un crecimiento equilibrado en el período 20082016 (tabla 5).

\subsection{ANÁLISIS ECONÓMICO}

Las empresas integrantes de BUSMET, a pesar de que los ingresos de explotación han disminuido un 7\% en el período analizado, han incrementado su resultado neto en este período, y presentan un valor añadido positivo (diferencia entre ingresos de explotación y gastos de explotación) que aumenta año tras año, en sintonía con la productividad del personal y los resultados. En consecuencia, presentan buenas rentabilidades, a nivel financiero y económico, es decir, la ROE (en inglés Return On Equity), como utilidades que reciben los inversionistas es buena, y la ROA (en inglés Return On Assets), como grado de eficiencia de la empresa en el uso de sus recursos económicos o activos para la obtención de beneficios, también es buena. 
Tabla 6. Análisis económico de las empresas integrantes de Busmet

\begin{tabular}{|c|c|c|c|c|c|c|c|c|c|}
\hline $\begin{array}{c}\text { Miles de } \\
\text { Euros }\end{array}$ & 2008 & 2009 & 2010 & 2011 & 2012 & 2013 & 2014 & 2015 & 2016 \\
\hline $\begin{array}{l}\text { Ingre- } \\
\text { sos de } \\
\text { explota- } \\
\text { ción }\end{array}$ & 8857,16 & 8002,41 & 9152,38 & 9311,01 & 9350,55 & 9234,66 & 9631,86 & 9719,87 & 8186,41 \\
\hline $\begin{array}{c}\text { Gastos } \\
\text { de per- } \\
\text { sonal }\end{array}$ & 3817,53 & 3485,10 & 3845,79 & 4023,21 & 4064,87 & 4023,75 & 4124,52 & 4270,90 & 3517,51 \\
\hline BAIT & 403,33 & 626,69 & 926,05 & 980,22 & 790,35 & 807,86 & 1341,94 & 1394,92 & 1080,84 \\
\hline $\begin{array}{l}\text { Gastos } \\
\text { Finan- } \\
\text { cieros }\end{array}$ & 258,01 & 180,52 & 159,76 & 189,33 & 191,08 & 193,33 & 253,53 & 215,45 & 62,02 \\
\hline $\begin{array}{l}\text { Resulta- } \\
\text { do neto }\end{array}$ & 140,47 & 362,49 & 590,93 & 638,87 & 481,99 & 481,09 & 779,47 & 873,33 & 787,83 \\
\hline $\mathrm{ROA}$ & $4,28 \%$ & $5,73 \%$ & $8,07 \%$ & $8,62 \%$ & $7,28 \%$ & $7,38 \%$ & $11,01 \%$ & $11,18 \%$ & $13,07 \%$ \\
\hline $\mathrm{ROE}$ & $4,82 \%$ & $9,63 \%$ & $13,94 \%$ & $14,08 \%$ & $9,92 \%$ & $9,20 \%$ & $13,18 \%$ & $13,26 \%$ & $15,98 \%$ \\
\hline $\begin{array}{l}\text { Gastos } \\
\text { Finan- } \\
\text { cieros/ } \\
\text { ventas }\end{array}$ & $2,91 \%$ & $2,26 \%$ & $1,75 \%$ & $2,03 \%$ & $2,04 \%$ & $2,09 \%$ & $2,45 \%$ & $2,22 \%$ & $0,76 \%$ \\
\hline $\begin{array}{c}\text { Valor } \\
\text { añadido }\end{array}$ & 403,33 & 626,69 & 926,05 & 980,22 & 790,35 & 807,86 & 1341,94 & 1394,92 & 1080,84 \\
\hline $\begin{array}{l}\text { Produc- } \\
\text { tividad } \\
\text { de per- } \\
\text { sonal }\end{array}$ & $10,57 \%$ & $17,98 \%$ & $24,08 \%$ & $24,36 \%$ & $19,44 \%$ & $20,08 \%$ & $32,54 \%$ & $32,66 \%$ & $30,73 \%$ \\
\hline
\end{tabular}

De otra parte, la ROE>ROA, de manera que, el endeudamiento no perjudica a estas empresas, cabe resaltar, que el endeudamiento ha disminuido en el período analizado y consecuentemente también los gastos financieros. Por tanto, las empresas integrantes de BUSMET presentan una buena situación económica (tabla 6).

\subsection{ANÁLISIS PATRIMONIAL}

Las empresas integrantes de BUSMET han experimentado una capitalización en el período objeto de estudio, ya que han aumentado el patrimonio neto en un 69\%, de manera que presentan más riqueza y garantías para con terceros. Esta capitalización se justifica por incrementos en el resultado neto (RN) y resultado global (RG) en el período objeto de estudio (tabla 7). 
Tabla 7. Análisis patrimonial de las empresas integrantes de Busmet

\begin{tabular}{|c|c|c|c|c|c|c|c|c|c|}
\hline MILES EUROS & 2008 & 2009 & 2010 & 2011 & 2012 & 2013 & 2014 & 2015 & 2016 \\
\hline RN & 140,47 & 362,49 & 590,93 & 638,87 & 481,99 & 481,09 & 779,47 & 873,33 & 787,83 \\
\hline RG & 158,12 & 394,56 & 595,24 & 602,63 & 467,27 & 481,77 & 753,29 & 826,05 & 853,76 \\
\hline $\begin{array}{l}\text { Patrimonio } \\
\text { Neto }\end{array}$ & $2.915,76$ & $3.765,41$ & $4.240,04$ & $4.537,90$ & $4.859,18$ & $5.228,56$ & $5.912,54$ & $6.584,42$ & $4.930,07$ \\
\hline
\end{tabular}

Tabla 8. Análisis económico y financiero de las empresas integrantes de Busmet

\begin{tabular}{|c|c|c|c|c|c|c|c|}
\hline $\begin{array}{c}\text { Período } \\
2008-2016\end{array}$ & $\begin{array}{c}\text { Fondo } \\
\text { Maniobra }\end{array}$ & Solvencia & $\begin{array}{c}\text { Endeuda- } \\
\text { miento }\end{array}$ & Facturación & ROE & ROA & Capitalización \\
\hline $\begin{array}{c}\text { Empresas } \\
\text { Busmet }\end{array}$ & Positivo & Buena & Normal & $\begin{array}{c}\text { Descenso } \\
7 \%\end{array}$ & $15,98 \%$ & $13,07 \%$ & $69 \%$ \\
\hline
\end{tabular}

\subsection{RESULTADOS E INTERPRETACIÓN}

Después de realizar el análisis económico y financiero y para hacer más representativo es estudio se presenta una tabla resumen (tabla 8) en la que se refleja el estudio de las 30 empresas integrantes de BUSMET (Tabla 8).

Las empresas integrantes de BUSMET presentan un crecimiento equilibrado en el período analizado. Son empresas con solvencia a corto plazo, con un endeudamiento normal y que presentan altas rentabilidades a pesar de que facturan un $7 \%$ menos y se han capitalizado en el período analizado. Son empresas que con las oportunas operaciones mercantiles entre el sector del transporte de autobuses han conseguido una salud económica financiera aceptable y en definitiva su supervivencia en el sector.

\section{ANÁLISIS DE COMUNICACIÓN WEB DE LA RSC EN BUSMET: CONTENIDO Y NAVEGABILIDAD}

En la ficha de recogida de los datos, el primer punto de análisis, es el que hace referencia al de la información general de las empresas de Busmet, en concreto, se analizan los idiomas en los que está traducida la página web, y se detecta que, a mayor tamaño mayor, más son los idiomas en que se traduce la página. Seguidamente, el análisis de contenido en el apartado "about us" da luz sobre los aspectos de identidad y cultura corporativa de la empresa. En referencia a la RSC, se analizan sus tres dimensiones: medioambiental, social y económica. Después del análisis se constata que las empresas hacen una buena comunicación sobre su historia, su misión, su visión, sus valores y su cultura de empresa.

Respecto al apartado correspondiente de RSC las empresas BUSMET reflejan las preocupaciones mediambientales, de calidad, de seguridad y sociales. 
En cuanto a la Navegabilidad losmapa webs y el buscador interno, en la mayoría de empresas BUSMET, están visibles y accesibles en sus páginas webs.

Por tanto, el análisis del contenido y la navegabilidad de los websites corporativos de las empresas integrantes en BUSMET, mediante análisis de sus webs (con una puntuación creciente de 1 a 5), contempla los elementos esenciales de la identidad corporativa, la información sobre RSC, la dimensión social y buscadores internos para los usuarios, entre otros aspectos.

Del estudio exploratorio se visibiliza la transparencia en estos ámbitos.

\subsection{ANÁLISIS DE CONTENIDO}

Para el análisis del contenido de la RSC se han identificado las siguientes variables (tabla 9):

La sección "About us" tiene una importancia fundamental en el proceso de comunicación de las empresas, ya que explica a los usuarios los elementos esenciales de la identidad corporativa, como los valores empresariales y la filosofía de la empresa, así como los fundamentos de la estrategia: misión, visión y valores.

La declaración de valores, suele ser el punto de partida para las políticas de RSC, como la igualdad, la transparencia y otros aspectos de la gobernanza.

En la tabla 10 se muestra la frecuencia de la variable "About us" y el 70\% de las empresas obtienen una puntuación mínima de 3 en los diferentes elementos, es decir, cumplen con la información corporativa (Tabla 10).

Respecto a la variable RSC, como apartado específico en el web corporativo, sólo cinco empresas (25\%) disponen de apartado propio. Y, la mayoría de empresas presenta alguna información, aunque dispersa en diferentes apartados.
Un 35\% de las empresas disponen de como mínimo dos dimensiones de la RSC y las empresas restantes, la mayoría no dispone de ningún ámbito. El contenido más frecuente de RSC, es en el ámbito medioambiental, para las empresas BUSMET, y la mayoría de información se refiere a normativas y legislaciones medioambientales, así como certificados medioambientales y de calidad.

En relación a la dimensión social, los websites corporativos privilegian la información al cliente-empresa ofreciéndoles información de atención al viajero. Tan sólo 6 empresas ofrecen información sobre los recursos humanos de la empresa. Once empresas utilizan las redes sociales (el 85,3\% no ofrecen interacción en estas redes desde el web corporativo), $y$, Twitter $y$ Facebook son las redes más utilizadas (55,0\%).

\subsection{ANÁLISIS DE NAVEGABILIDAD}

Para este análisis se han identificado las variables que se detallan en la tabla 11.

El análisis de las variables del Mapa Web revela que un 50\% de las empresas alcanzan una puntuación máxima en la variable "información principal" y un 20\% obtienen la puntuación mínima, a diferencia de la variable definida como "información de interés", ya que, el 85\% de los websites corporativos ofrecen abundantes herramientas para el usuario (FAQs, noticias, artículos, horarios etc.).

El contenido informativo general (no específico del Mapa Web) suele estar distribuido en diversos apartados, aunque disponen de "buscador interno", y el análisis de frecuencia revela que el $50 \%$ de las empresas de nuestro estudio ofrecen opciones eficientes de búsqueda para los clientes o usuarios. 
Tabla 9. Elementos de Contenido: About Us y Contenido

\begin{tabular}{|c|c|c|c|c|c|}
\hline \multicolumn{6}{|c|}{ CONTENIDO } \\
\hline \multicolumn{2}{|l|}{ Sección "ABOUT US" } & \multicolumn{4}{|c|}{ Apartado Específico de RSC } \\
\hline Elementos corporativo & Puntos & Apartado específico & Puntos & Dimensiones de la RSC & Puntos \\
\hline $\begin{array}{l}\text { Incluye elementos de identidad } \\
\text { corporativa }\end{array}$ & 5 & SI & 1 & $\begin{array}{l}\text { Todas dimensiones de } \\
\text { la RSC }\end{array}$ & 5 \\
\hline $\mathrm{H}^{\mathrm{a}} \mathrm{y}$ organigrama & 4 & NO & 0 & $\begin{array}{l}\text { Dispone de dos dimen- } \\
\text { siones de la RSC. }\end{array}$ & 4 \\
\hline $\begin{array}{c}\text { Presentación general de la em- } \\
\text { presa }\end{array}$ & 3 & & & $\begin{array}{l}\text { Dispone de una dimen- } \\
\text { sión de }\end{array}$ & 3 \\
\hline $\begin{array}{c}\text { NO dispone de ABOUT US pero } \\
\text { hace referencia a la identidad } \\
\text { (presentación) }\end{array}$ & 2 & & & $\begin{array}{l}\text { No dispone de pestaña } \\
\text { RSC pero la menciona } \\
\text { en la presentación }\end{array}$ & 2 \\
\hline NO dispone & 1 & & & No dispone & 1 \\
\hline
\end{tabular}

Tabla 10. Tabla de Frecuencia de la variable "About Us"

\begin{tabular}{|c|c|c|c|}
\hline Elementos corporativos & 0 & 0 & \\
\hline No dispone de ABOUT US & 1 & 2 & $10 \%$ \\
\hline No operativa & 2 & 4 & $20 \%$ \\
\hline No dispone de ABOUT US pero identidad en presentación & 3 & 2 & $10 \%$ \\
\hline Presentación general de la empresa & 4 & 4 & $20 \%$ \\
\hline Historia detallada y organigrama & 5 & 8 & $40 \%$ \\
\hline Incluye Identidad: Visión, Misión y Valores & & 20 & $100 \%$ \\
\hline TOTAL & & & \\
\hline
\end{tabular}

Tabla 10. Tabla de Frecuencia de la variable "About Us"

\begin{tabular}{|c|c|c|c|c|}
\hline \multicolumn{5}{|c|}{ NAVEGABILIDAD } \\
\hline & MAPA & WEB & BUSCADOR INTERNO & \\
\hline Información principal & Puntos & $\begin{array}{l}\text { Información de inte- } \\
\text { rés. FAQ's, Comenta- } \\
\text { rios, Ayuda usuario, } \\
\text { Contenidos y actuali- } \\
\text { zaciones }\end{array}$ & Buscador interno & Puntos \\
\hline Mapa web en el Lay Out & 5 & SI & Esta en Lay Out & 5 \\
\hline
\end{tabular}




\begin{tabular}{|c|c|c|c|c|c|}
\hline $\begin{array}{c}\text { Ambas aplicaciones siem- } \\
\text { pre visibles ó en el Menú }\end{array}$ & 4 & NO & 0 & Está siempre visible & 4 \\
\hline Mapa Web en la pàg. & 3 & & & Accesible desde la Home & 3 \\
\hline Indica Ruta de Navegación & 2 & & & Está en alguna sección & 2 \\
\hline No operativo & 1 & & & No operativo & 1 \\
\hline No dispone & 0 & & No dispone & 0 \\
\hline
\end{tabular}

\section{CONCLUSIONES}

Las ventajas de la propuesta de investigación derivan de la relevancia del sector del transporte, por su gran importancia en la actualidad, a nivel del desarrollo económico que representa y el impacto en medioambiente que implica. Se trata de un sector sujeto a importantes retos económicos y medioambientales.

Mediante la metodología exploratoria utilizada (análisis económico y financiero y análisis de la transparencia web), se evidencia que la integración de las operadoras privadas de transporte de viajeros en autobús (gracias el oportuno plan estratégico de comunicación), como en el caso BUSMET, permite asegurar la salud económica financiera de las empresas integrantes y consigue incrementar su cuota de mercado, avalando la supervivencia en el sector, por el crecimiento equilibrado y los aceptables indicadores del modelo de cohesión empresarial.

De otra parte, las empresas integrantes de BUSMET, a nivel de transparencia web, comunican de manera discreta la problemática de la sostenibilidad, es decir, en sus webs las políticas de RSC deben incrementar en contenido y comunicación dada la trascendencia medioambiental que representa este sector. Cabe destacar que un 70\% de las empresas integrantes de BUSMET cumplen los ítems del contenido en referencia a la variable "About us", en cambio respecto a la variable RSC sólo un 25\%-35\% disponen de dicha información, referida especialmente al ámbito medioambiental (normativas y certificados de calidad). La variable de atención al pasajero, dispone de una correcta transparencia a diferencia de la variable de recursos humanos. Finalmente, resaltar que muchas de estas empresas utilizan las redes sociales.

Respecto a la navegabilidad de las variables del Mapa Web un 50\% de las empresas son transparentes en la información principal y respecto a la información de interés, el $85 \%$ de los websites corporativos ofrecen abundantes herramientas para el usuario (FAQs, noticias, artículos, horarios etc.). El contenido informativo general está distribuido en diversos apartados, con un buscador interno, para los usuarios, en un $50 \%$ de los casos.

Respecto a la parte técnica de la metodología utilizada cabe destacar, que a nivel del análisis económico y financiero, se han utilizado los indicadores de referencia contemplados en la literatura académica; y respecto al análisis de la transparencia web se ha utilizado una ficha técnica de recogida y sistematización de la información cualitativa y cuantitativa utilizada en publicaciones académicas (Aramayo et al. 2016). La plantilla de analisis tiene la finalidad de registrar y tratar la información referente a 
más de 30 entradas de las diversas variables de cada una de las empresas de Busmet. Los ejes son 2, el Analisis de Contenido, referido a los elementos corporativos de la RSC, sobre sus tres ejes o dimensiones. Para medir la categoria "Navegabilidad" se han estudiado los datos contenidos en el mapa web, las de uso general por parte del usuario y las opciones del buscador web. El sistema de puntuación va de un mínimo de 0 a 5 como tope.

A modo de conclusión final es destacable que el modelo BUSMET, en el mapa del transporte de viajeros en la zona metropolitana de BarceIona, evidencia la supervivencia de las empresas integrantes por sus correctos indicadores económicos y financieros en el período 20082016, junto con la adecuada trasparencia en su comunicación web, aunque la variable RSC debe gozar de mayor transparencia en los webs. BUSMET se consolida como modelo que busca mediante la cohesión empresarial, la calidad del servicio para conseguir las oportunas subvenciones del transporte de pasajeros, con previsiones de crecimiento en un futuro próximo. Su comunicación web y su transparencia, es correcta a nivel de contenido, con una aceptable navegabilidad, aunque debe desarrollar y comunicar oportunamente más políticas de RSC, especialmente en el ámbito medioambiental, por los grandes cambios en este ámbito en la logística y el transporte.
La comunicación web se corresponde al tamaño de las empresas, cuanto mayor es la empresa, mayor proporcionalmente es la comunicación y contenidos en las páginas webs. Y a la inversa, cuanto menor es la empresa, más escasa es la comunicación y conenidos disponibles. Al igual sucede con las comunicaciones de las acciones de RSC, las empresas más pequeñas suelen realizar menos acciones de RSC. Se contempla, en un futuro, la creación de un manual de buenas prácticas para unificar e internalizar la RSC en las empresas que conforman Busmet. 


\section{BIBLIOGRAFÍA}

Araque, R., y Montero, M. (2006). La responsabilidad social de la empresa a debate. Barcelona: Icaria.

Aramayo, A., Arimany, N., Uribe, C., Sabata, A. (2016). Web communication of CSR and financial performance: A study of catalan meat companies. Intangible Capital, 12(2), 391-419. http://dx.doi.org/10.3926/ic.590

Cameron, W. (2004). Public accountability: effectiveness, equity, ethics. Australian Journal of Public Administration, 63(4), 59-67. doi:10.1111/ j.1467-8500.2004.00402.x

Cilliberti, F., Pontradolfo, P. y Scozzi, B. (2008). Investigating Corporate Social responsibility in supply chains: a SME perspective. Journal of cleaner production, 16 (15), 1579-1588. doi.org/10.1016/j.jclepro.2008.04.016

Currás, R., Bigne, E. y Alvarado, A. (2009). The role of self-definitional principles inconsumer identification with a socially responsible company. Journal of Business Ethics, 89, 547-564. doi.org/10.1007/s10551-0080016-6

De la Cuesta, M. (2004, septiembre 5). El porqué de la responsabilidad social corporativa. Boletín Económico ICE, (2813). Recuperado 27 noviembre 2017, de http://www.revistasice.com/CachePDF/BICE_2813_45-58_3878E11FE5EA92486445FF5860C4DF47.pdf

De Rus, G. y Nombela, G. (1997). Privatisation of Urban Bus Services in Spain. Journal of transport economics and Policy, 31 (1), 115-129.

Diane Mollenkopf, Hannah Stolze, Wendy L. Tate y Monique Ueltschy, (2010). Green, lean, and global supply chains. International Journal of Physical Distribution \& Logistics Management, 40,1/2, 14-41. doi. org/10.1108/09600031011018028

Huertas, A., y Xifra J. (2009). ¿Marcas o genéricos? La comunicación en línea de las marcas farmacéuticas. Revista de Estudios de comunicación, 27, 251-270.

Jonathan W. Palmer (2002). Web Site Usability, Design, and Performance Metrics. Information Systems Research,13:2,151-167.

Martin Urbano, P., Ruiz, A. y Sanchez, J.I. (2012). El sistema de transporte público en España: Una perspectiva interregional. Cuadernos de Economía, 31 (58), 195-228. 
Meijer, A. J. (2007). Publishing public performance results on the internet. Do stakeholders use the internet to hold Dutch public service organizations to account? Government Information Quarterly, 24(1), 165-185. doi.org/10.1016/j.giq.2006.01.014

Piñeiro, P., Quintas, M. A. y Caballero, G. (2009). Incidencia de la proactividad medioambiental en el rendimiento de las empresas constructoras españolas. Revista europea de dirección y economía de la empresa, 18(2), 79-106.

Porter, M. E. y Kramer, M. R. (2003). La filantropía empresarial como ventaja competitiva. Harvard Deusto Business Review, (112), 7-20.

Rindova, V. P., Wiliiamson, I. O., Petkova, A. P. y Sever, J. M. (2005). Being good or being known: an empirical examination of the dimensions, antecedents, and consequences of organizational reputation. Academy of Management Journal, 48(6),1033-1049. doi:10.5465/AMJ.2005.19573108

Robbins, S. P. (1994). Comportamiento organizacional. México: Prentice Hall.

Rodríguez, F. y García de Madariaga, J. (2011). Responsabilidad social corporativa y gobierno corporativo: impacto sobre la reputación de las compañías del sector automovilístico y la satisfacción del cliente. Revista de la Responsabilidad Social Empresarial, 7 (3,1), 47-104.

Rodriguez, M. A., Ricart, J. E. y Sanchez, P. (2002), Sustainable Development and the Sustainability of Competitive Advantage: A Dynamic and Sustainable View of the Firm. Creativity and Innovation Management, 11: 135-146. doi: 10.1111/1467-8691.00246

Saxton, T. y Dollinger, M. (2004). Target reputation and appropriability: picking and deploying resources in acquisitions. Journal of Management, 30(1), 123-147. doi:org/10.1016/j.jm.2003.01.006

Tagesson, T., Blank, V., Broberg, P. y Collin, S. (2009). What explains the extent and content of social and environmental disclosures on corporate websites: a study of social and environmental reporting in Swedish listed corporations? Corp. Soc.Responsib. Environ. Management, 16, 352-364. doi:10.1002/csr.194 